\title{
REMARKS ON SQUARE FUNCTIONS IN THE LITTLEWOOD-PALEY THEORY
}

\author{
Shuichi Sato
}

\begin{abstract}
We prove that certain square function operators in the Littlewood-Paley theory defined by the kernels without any regularity are bounded on $L_{w}^{p}, 1<p<\infty$, $w \in A_{p}$ (the weights of Muckenhoupt). Then, we give some applications to the Carleson measures on the upper half space.
\end{abstract}

\section{INTRODUCTION}

In this note we shall prove weighted $L^{p}$-estimates for the Littlewood-Paley type square functions arising from kernels satisfying only size and cancellation conditions. Suppose that $\psi \in L^{1}\left(\mathbf{R}^{n}\right)$ satisfies

$$
\int_{\mathbf{R}^{n}} \psi(x) d x=0
$$

We consider a square function of Littlewood-Paley type

$$
S(f)(x)=S_{\psi}(f)(x)=\left(\int_{0}^{\infty}\left|\psi_{t} \star f(x)\right|^{2} \frac{d t}{t}\right)^{1 / 2}
$$

where $\psi_{t}(x)=t^{-n} \psi\left(t^{-1} x\right)$.

If $\psi$ satisfies, in addition to (1.1),

$$
\begin{aligned}
|\psi(x)| \leqslant c(1+|x|)^{-n-\varepsilon} \text { for some } & \varepsilon>0 \\
\int_{\mathbf{R}^{n}}|\psi(x-y)-\psi(x)| d x \leqslant c|y|^{\varepsilon} \text { for some } & \varepsilon>0
\end{aligned}
$$

then it is known that the operator $S$ is bounded on $L^{p}\left(\mathbf{R}^{n}\right)$ for all $p \in(1, \infty)$ (see Benedek, Calderón and Panzone [1]). Well-known examples are as follows.

EXAMPLE 1: Let $P_{t}(x)$ be the Poisson kernel for the upper half space $\mathbf{R}^{n} \times(0, \infty)$ :

$$
P_{t}(x)=c_{n} \frac{t}{\left(|x|^{2}+t^{2}\right)^{(n+1) / 2}}
$$

Received 22nd December, 1997

Copyright Clearance Centre, Inc. Serial-fee code: 0004-9729/98 \$A2.00+0.00. 
Put

$$
\psi(x)=\left(\frac{\partial}{\partial t} P_{t}(x)\right)_{t=1}
$$

Then $S_{\psi}(f)$ is the Littlewood-Paley $g$ function.

EXAMPLE 2: Consider the Haar function $\psi$ on $\mathbf{R}$ :

$$
\psi(x)=\chi_{[-1,0]}(x)-\chi_{[0,1]}(x)
$$

where $\chi_{E}$ denotes the characteristic function of a set $E$. Then, $S_{\psi}(f)$ is the Marcinkiewicz integral

$$
\mu(f)(x)=\left(\int_{0}^{\infty}|F(x+t)+F(x-t)-2 F(x)|^{2} \frac{d t}{t^{3}}\right)^{1 / 2}
$$

where $F(x)=\int_{0}^{x} f(y) d y$.

In this note, we shall prove that the $L^{p}$-boundedness of $S$ still holds without the assumption (1.3); the conditions (1.1) and (1.2) only are sufficient. This is already known for the $L^{2}$-case (see Coifman and Meyer [3, p.148], and also Journé [7, pp. 81-82] for a proof).

To state our result more precisely, we consider the least non-increasing radial majorant of $\psi$

$$
h_{\psi}(|x|)=\sup _{|y| \geqslant|x|}|\psi(y)|
$$

We also need to consider two seminorms

$$
\begin{gathered}
B_{\varepsilon}(\psi)=\int_{|x|>1}|\psi(x)||x|^{\varepsilon} d x \quad \text { for } \quad \varepsilon>0, \\
D_{u}(\psi)=\left(\int_{|x|<1}|\psi(x)|^{u} d x\right)^{1 / u} \quad \text { for } \quad u>1 .
\end{gathered}
$$

We shall prove the following result.

Theorem 1. Put $H_{\psi}(x)=h_{\psi}(|x|)$. If $\psi \in L^{1}\left(\mathbf{R}^{n}\right)$ satisfies (1.1) and

(1) $B_{\varepsilon}(\psi)<\infty \quad$ for some $\varepsilon>0$;

(2) $D_{u}(\psi)<\infty$ for some $u>1$;

(3) $H_{\psi} \in L^{1}\left(\mathbf{R}^{n}\right)$;

then the operator $S_{\psi}$ is bounded on $L_{w}^{p}$ :

$$
\left\|S_{\psi}(f)\right\|_{L_{w}^{p}} \leqslant C_{p, w}\|f\|_{L_{w}^{p}}
$$


for all $p \in(1, \infty)$ and $w \in A_{p}$, where $A_{p}$ denotes the weight class of Muckenhoupt (see $[6,7])$, and

$$
\|f\|_{L_{w}^{p}}=\|f\|_{L^{p}(w)}=\left(\int_{\mathbf{R}^{n}}|f(x)|^{p} w(x) d x\right)^{1 / p}
$$

In fact, we shall prove a more general result.

ThEOREM 2. Suppose that $\psi \in L^{1}\left(\mathbf{R}^{n}\right)$ satisfies (1.1) and

(1) $B_{\varepsilon}(\psi)<\infty \quad$ for some $\varepsilon>0$;

(2) $D_{u}(\psi)<\infty$ for some $u>1$;

(3) $|\psi(x)| \leqslant h(|x|) \Omega\left(x^{\prime}\right) \quad\left(x^{\prime}=|x|^{-1} x\right)$ for some non-negative functions $h$ and $\Omega$ such that

(a) $h(r)$ is non-increasing for $r \in(0, \infty)$;

(b) if $H(x)=h(|x|), H \in L^{1}\left(\mathbf{R}^{n}\right)$;

(c) $\Omega \in L^{q}\left(S^{n-1}\right)$ for some $q, 2 \leqslant q \leqslant \infty$.

Then, the operator $S_{\psi}$ is bounded on $L_{w}^{p}$ for $p>q^{\prime}$ and $w \in A_{p / q^{\prime}}$, where $q^{\prime}$ denotes the conjugate exponent of $q$.

When $\psi$ is compactly supported, we have another formulation, which is not included in Theorem 2.

TheOREM 3. Suppose that $\psi \in L^{1}\left(\mathbf{R}^{n}\right)$ satisfies (1.1) and

(1) $\psi$ is compactly supported ;

(2) $\psi \in L^{q}\left(\mathbf{R}^{n}\right)$ for some $q \geqslant 2$.

Then $S_{\psi}: L_{w}^{p} \rightarrow L_{w}^{p}$ for $p>q^{\prime}$ and $w \in A_{p / q^{\prime}}$.

These results will be derived from more abstract ones. Let $\psi \in L^{1}\left(\mathbf{R}^{n}\right)$ satisfy (1.1). We also assume the following :

(1) There exists $\varepsilon \in(0,1)$ such that

$$
\int_{1}^{2}|\widehat{\psi}(t \xi)|^{2} d t \leqslant c \min \left(|\xi|^{\varepsilon},|\xi|^{-\varepsilon}\right) \quad \text { for all } \quad \xi \in \mathbf{R}^{n}
$$

where $\widehat{\psi}$ denotes the Fourier transform

$$
\left.\widehat{\psi}(\xi)=\int \psi(x) e^{-2 \pi i(x, \xi)} d x, \quad\langle x, \xi\rangle=\sum_{j=1}^{n} x_{j} \xi_{j} \quad \text { (the inner product in } \mathbf{R}^{n}\right) .
$$

(2) Let $1 \leqslant s \leqslant 2$. For all $w \in A_{s}$, we have

$$
\sup _{k \in \mathbf{Z}} \int_{\mathbf{R}^{n}} \int_{1}^{2}\left|\psi_{t 2^{k}} \star f(x)\right|^{2} d t w(x) d x \leqslant C_{w}\|f\|_{L_{w}^{2}}^{2} \quad \text { for all } \quad f \in \mathcal{S}\left(\mathbf{R}^{n}\right) \text {, }
$$

where $\mathbf{Z}$ denotes the integer group and $\mathcal{S}\left(\mathbf{R}^{n}\right)$ the Schwartz space. 
Under these assumptions the following holds.

Proposition 1. For $p>2 / s$ and $w \in A_{p s / 2}$, the operator $S_{\psi}$ is bounded on $L_{w}^{p}$.

This will be used to prove the next result.

Proposition 2. Put

$$
J_{\varepsilon}(\psi)=\sup _{|\xi|=1} \iint_{\mathbf{R}^{n} \times \mathbf{R}^{n}}|\psi(x) \psi(y)||\langle\xi, x-y\rangle|^{-\varepsilon} d x d y \quad \text { for } \quad \varepsilon \in(0,1] .
$$

Let $\psi \in L^{1}$ satisfy (1.1) and (1.5). Then if $B_{\varepsilon}(\psi)<\infty$ and $J_{\varepsilon}(\psi)<\infty$ for some $\varepsilon \in(0,1]$, the operator $S_{\psi}$ is bounded on $L_{w}^{p}$ for $p>2 / s$ and $w \in A_{p s / 2}$.

In Section 2, we shall prove Proposition 1 by the method of the proof of Duoandikoetxea and Rubio de Francia [5, Corollary 4.2] and then Proposition 2 by using Proposition 1. Proposition 2 will be applied to prove Theorems 2 and 3 in Section 3. Finally, in Section 4, we shall give some applications of Theorem 1 to generalised Marcinkiewicz integrals and the Carleson measures on the upper half space $\mathbf{R}^{n} \times(0, \infty)$.

To conclude this section, we state a result for the $L^{2}$-case, from which the result of Coifman-Meyer mentioned above immediately follows, and the idea of the proof will be applied later too (see the proof of Lemma 2).

Proposition 3. Suppose that $\psi \in L^{1}$ satisfies (1.1). Let

$$
L(\psi)=\sup _{|\xi|=1} \iint_{\mathbf{R}^{n} \times \mathbf{R}^{n}}|\psi(x) \psi(y)||\log |\langle\xi, x-y\rangle|| d x d y .
$$

Then, if $L(\psi)<\infty$, the operator $S_{\psi}$ is bounded on $L^{2}$.

Proof: It is sufficient to show that

$$
\sup _{|\xi|=1} \int_{0}^{\infty}|\widehat{\psi}(t \xi)|^{2} \frac{d t}{t}<\infty
$$

We write

$$
|\widehat{\psi}(t \xi)|^{2}=\widehat{\psi}(t \xi) \overline{\widehat{\psi}(t \xi)}=\iint_{\mathbf{R}^{n} \times \mathbf{R}^{n}} \psi(x) \overline{\psi(y)} e^{-2 \pi i t\langle\xi, x-y\rangle} d x d y
$$

and so

$$
\int_{0}^{\infty}|\widehat{\psi}(t \xi)|^{2} \frac{d t}{t}=\lim _{N \rightarrow \infty, \varepsilon \rightarrow 0} \iint \psi(x) \overline{\psi(y)}\left(\int_{\varepsilon}^{N} e^{-2 \pi i t(\xi, x-y)} \frac{d t}{t}\right) d x d y .
$$


Note that

$$
\int_{\varepsilon}^{N}\left(e^{-2 \pi i t(\xi, x-y)}-\cos (2 \pi t)\right) \frac{d t}{t} \rightarrow-\log |\langle\xi, x-y\rangle|-i \frac{\pi}{2} \operatorname{sgn}\langle\xi, x-y\rangle
$$

as $N \rightarrow \infty$ and $\varepsilon \rightarrow 0$, and the integral is bounded, uniformly in $\varepsilon$ and $N$, by

$$
c(1+|\log |\langle\xi, x-y\rangle||)
$$

Thus, using (1.1) and the dominated convergence theorem, we get

$$
\int_{0}^{\infty}|\widehat{\psi}(t \xi)|^{2} \frac{d t}{t}=\iint \psi(x) \overline{\psi(y)}\left(-\log |\langle\xi, x-y\rangle|-i \frac{\pi}{2} \operatorname{sgn}\langle\xi, x-y\rangle\right) d x d y
$$

This immediately implies the conclusion.

REMARK. In the one-dimensional case, it is easy to see that if

$$
\int|\psi(x)| \log (2+|x|) d x<\infty \quad \text { and } \quad \int|\psi(x)| \log (2+|\psi(x)|) d x<\infty
$$

then $L(\psi)<\infty$, and so $S_{\psi}: L^{2} \rightarrow L^{2}$.

\section{Proofs of Propositions 1 AND 2}

We use a Littlewood-Paley decomposition. Let $f \in \mathcal{S}\left(\mathbf{R}^{n}\right)$, and define

$$
\widehat{\Delta_{j}(f)}(\xi)=\Psi\left(2^{j} \xi\right) \widehat{f}(\xi) \quad \text { for } \quad j \in \mathbf{Z}
$$

where $\Psi \in C^{\infty}$ is supported in $\{1 / 2 \leqslant|\xi| \leqslant 2\}$ and satisfies

$$
\sum_{j \in \mathbf{Z}} \Psi\left(2^{j} \xi\right)=1 \quad \text { for } \quad \xi \neq 0
$$

Decompose

$$
f \star \psi_{t}(x)=\sum_{j \in \mathbf{Z}} \sum_{k \in \mathbf{Z}} \Delta_{j+k}\left(f \star \psi_{t}\right)(x) \chi_{\left[2^{k}, 2^{k+1}\right)}(t)=\sum_{j \in \mathbf{Z}} F_{j}(x, t)
$$

say, and define

$$
T_{j}(f)(x)=\left(\int_{0}^{\infty}\left|F_{j}(x, t)\right|^{2} \frac{d t}{t}\right)^{1 / 2}
$$

Then

$$
S(f)(x) \leqslant \sum_{j \in \mathbf{Z}} T_{j}(f)(x) .
$$


Put $E_{j}=\left\{2^{-1-j} \leqslant|\xi| \leqslant 2^{1-j}\right\}$. Then by the Plancherel theorem and (1.4) we have

$$
\begin{aligned}
\left\|T_{j}(f)\right\|_{2}^{2} & =\sum_{k \in \mathbf{Z}} \int_{\mathbf{R}^{n}} \int_{2^{k}}^{2^{k+1}}\left|\Delta_{j+k}\left(f \star \psi_{t}\right)(x)\right|^{2} \frac{d t}{t} d x \\
& \leqslant \sum_{k \in \mathbf{Z}} c \int_{E_{j+k}}\left(\int_{2^{k}}^{2^{k+1}}|\widehat{\psi}(t \xi)|^{2} \frac{d t}{t}\right)|\widehat{f}(\xi)|^{2} d \xi \\
& \leqslant \sum_{k \in \mathbf{Z}} c \int_{E_{j+k}} \min \left(\left|2^{k} \xi\right|^{\varepsilon},\left|2^{k} \xi\right|^{-\varepsilon}\right)|\widehat{f}(\xi)|^{2} d \xi \\
& \leqslant c 2^{-\varepsilon|j|} \sum_{k \in \mathbf{Z}} \int_{E_{j+k}}|\widehat{f}(\xi)|^{2} d \xi \\
& \leqslant c 2^{-\varepsilon|j|}\|f\|_{2}^{2},
\end{aligned}
$$

where the last inequality holds since the sets $E_{j}$ are finitely overlapping. (We denote by $\|\cdot\|_{p}$ the ordinary $L^{p}$-norm.)

On the other hand, for $w \in A_{s}$ by (1.5) we see that

$$
\begin{aligned}
\left\|T_{j}(f)\right\|_{L_{w}^{2}}^{2} & =\sum_{k \in \mathbf{Z}} \int_{\mathbf{R}^{n}} \int_{2^{k}}^{2^{k+1}}\left|\Delta_{j+k}(f) \star \psi_{t}(x)\right|^{2} \frac{d t}{t} w(x) d x \\
& \leqslant \sum_{k \in \mathbf{Z}} c \int_{\mathbf{R}^{n}}\left|\Delta_{j+k}(f)(x)\right|^{2} w(x) d x \\
& \leqslant c\|f\|_{L_{w}^{2}}^{2},
\end{aligned}
$$

where the last inequality follows from a well-known Littlewood-Paley inequality for $L_{w}^{2}$ since $A_{s} \subset A_{2}$.

Interpolating with change of measures between the two estimates above, we get

$$
\left\|T_{j}(f)\right\|_{L^{2}\left(w^{u}\right)} \leqslant c 2^{-\varepsilon(1-u)|j| / 2}\|f\|_{L^{2}\left(w^{u}\right)}
$$

for $u \in(0,1)$. If we choose $u$ (close to 1 ) so that $w^{1 / u} \in A_{s}$, then from this inequality we get

$$
\left\|T_{j}(f)\right\|_{L_{w}^{2}} \leqslant c 2^{-\varepsilon(1-u)|j| / 2}\|f\|_{L_{w}^{2}}
$$

and so

$$
\|S(f)\|_{L_{w}^{2}} \leqslant \sum_{j \in \mathbf{Z}}\left\|T_{j}(f)\right\|_{L_{w}^{2}} \leqslant c\|f\|_{L_{w}^{2}} .
$$

Thus the extrapolation theorem of Rubio de Francia [8] implies the conclusion.

To derive Proposition 2 from Proposition 1 we need the following lemmas. 
LEMMA 1. If $\psi \in L^{1}\left(\mathbf{R}^{n}\right)$ satisfies (1.1) and $B_{\varepsilon}(\psi)<\infty$ for $\varepsilon \in(0,1]$, then

$$
|\widehat{\psi}(\xi)| \leqslant c|\xi|^{\varepsilon} \quad \text { for all } \quad \xi \in \mathbf{R}^{n} \text {. }
$$

Proof: Since $a \leqslant a^{\varepsilon}$ for $a, \varepsilon \in(0,1]$, we see that

$$
\begin{aligned}
|\widehat{\psi}(\xi)|=\left|\int \psi(x)\left(e^{-2 \pi i\langle x, \xi\rangle}-1\right) d x\right| & \leqslant c \int|\psi(x)| \min (1,|\langle x, \xi\rangle|) d x \\
& \leqslant c|\xi|^{\varepsilon} \int|\psi(x)||x|^{\varepsilon} d x
\end{aligned}
$$

This completes the proof.

LEMMA 2. If $\psi \in L^{1}\left(\mathbf{R}^{n}\right)$ and $J_{\varepsilon}(\psi)<\infty$ for $\varepsilon \in(0,1]$, then

$$
\int_{1}^{2}|\widehat{\psi}(t \xi)|^{2} d t \leqslant c|\xi|^{-\varepsilon} \quad \text { for all } \quad \xi \in \mathbf{R}^{n}
$$

Proof: As in the proof of Proposition 3, we see that

$$
\int_{1}^{2}|\widehat{\psi}(t \xi)|^{2} d t=\iint_{\mathbf{R}^{n} \times \mathbf{R}^{n}} \psi(x) \overline{\psi(y)} \frac{e^{-4 \pi i\langle\xi, x-y\rangle}-e^{-2 \pi i\langle\xi, x-y\rangle}}{-2 \pi i\langle\xi, x-y\rangle} d x d y .
$$

Thus

$$
\begin{aligned}
\int_{1}^{2}|\widehat{\psi}(t \xi)|^{2} d t & \leqslant c \iint_{\mathbf{R}^{n} \times \mathbf{R}^{n}}|\psi(x) \psi(y)| \min \left(1,|\langle\xi, x-y\rangle|^{-1}\right) d x d y \\
& \leqslant c J_{\varepsilon}(\psi)|\xi|^{-\varepsilon}
\end{aligned}
$$

This completes the proof.

Now, we can see that Proposition 1 implies Proposition 2, since the condition (1.4) follows from Lemmas 1 and 2 .

\section{Proofs of Theorems 2 and 3}

To get Theorem 2 from Proposition 2 we need Lemmas 3 and 4 below. First, we give a sufficient condition for $J_{\varepsilon}(\psi)<\infty$.

LEMMA 3. Let $h(r), h \geqslant 0$, be a non-increasing function for $r>0$ satisfying $H \in L^{1}\left(\mathbf{R}^{n}\right) \cap L^{\infty}\left(\mathbf{R}^{n}\right)$, where $H(x)=h(|x|)$, and let $\Omega \in L^{v}\left(S^{n-1}\right), v>1, \Omega \geqslant 0$. Suppose that $F$ is a non-negative function such that

$$
F(x) \leqslant h(|x|) \Omega\left(x^{\prime}\right) \quad \text { for } \quad|x|>1
$$


and $D_{u}(F)<\infty$ for $u>1$. Then $J_{\varepsilon}(F)<\infty$ if $\varepsilon<\min \left(1 / u^{\prime}, 1 / v^{\prime}\right)$.

Proof: For non-negative functions $f, g$ and $\xi \in S^{n-1}$ put

$$
L_{\varepsilon}(f, g ; \xi)=\iint_{\mathbf{R}^{n} \times \mathbf{R}^{n}} f(x) g(y)|\langle\xi, x-y\rangle|^{-\varepsilon} d x d y .
$$

Decompose $F$ as $F=E+G$, where $E(x)=F(x)$ if $|x|<1$ and $E(x)=0$ otherwise. Then

$$
L_{\varepsilon}(F, F ; \xi)=L_{\varepsilon}(E, E ; \xi)+2 L_{\varepsilon}(E, G ; \xi)+L_{\varepsilon}(G, G ; \xi) .
$$

We show that each of $L_{\varepsilon}(E, E ; \xi), L_{\varepsilon}(E, G ; \xi)$ and $L_{\varepsilon}(G, G ; \xi)$ is bounded by a constant independent of $\xi$ if $\varepsilon<\min \left(1 / u^{\prime}, 1 / v^{\prime}\right)$.

First, by Hölder's inequality and a change of variables

$$
L_{\varepsilon}(E, E ; \xi) \leqslant\|E\|_{u}^{2}\left(\iint_{|x|<1,|y|<1}\left|x_{1}-y_{1}\right|^{-\varepsilon u^{\prime}} d x d y\right)^{1 / u^{\prime}},
$$

where we note that $\|E\|_{u}=D_{u}(F)$.

Next, by Hölder's inequality again

$$
L_{\varepsilon}(E, G ; \xi) \leqslant\|E\|_{u}\left(\int_{|x|<1}\left(\int_{\mathbb{R}^{n}} G(y)\left|x_{1}-\langle\xi, y\rangle\right|^{-\varepsilon} d y\right)^{u^{\prime}} d x\right)^{1 / u^{\prime}} .
$$

For $s>0$, let

$$
I_{\varepsilon}(s)=\int_{S^{n-1}}\left|x_{1}-\langle\xi, s \omega\rangle\right|^{-\varepsilon} \Omega(\omega) d \sigma(\omega)
$$

for fixed $x_{1}$ and $\xi$, where $d \sigma$ denotes the Lebesgue surface measure of $S^{n-1}$ (when $n=1$, let $\sigma(\{1\})=\sigma(\{-1\})=1)$. Then by Hölder's inequality

$$
I_{\varepsilon}(s) \leqslant\left(N_{\varepsilon v^{\prime}}(s)\right)^{1 / v^{\prime}}\|\Omega\|_{v},
$$

where

$$
N_{\varepsilon}(s)=\int_{S^{n-1}}\left|x_{1}-s \omega_{1}\right|^{-\varepsilon} d \sigma(\omega) .
$$

Thus, using Hölder's inequality,

$$
\begin{aligned}
\int_{\mathbf{R}^{n}} G(y)\left|x_{1}-\langle\xi, y\rangle\right|^{-\varepsilon} d y & \leqslant \int_{0}^{\infty} h(s) s^{n-1} I_{\varepsilon}(s) d s \\
& \leqslant\|\Omega\|_{v} \int_{0}^{\infty} h(s) s^{n-1}\left(N_{\varepsilon v^{\prime}}(s)\right)^{1 / v^{\prime}} d s \\
& \leqslant c\|H\|_{1}^{1 / v}\|\Omega\|_{v}\left(\int_{0}^{\infty} h(s) s^{n-1} N_{\varepsilon v^{\prime}}(s) d s\right)^{1 / v^{\prime}} \\
& =c\|H\|_{1}^{1 / v}\|\Omega\|_{v}\left(\int_{\mathbf{R}^{n}} h(|y|)\left|x_{1}-y_{1}\right|^{-\varepsilon v^{\prime}} d y\right)^{1 / v^{\prime}}
\end{aligned}
$$


Therefore, the desired estimate for $L_{\varepsilon}(E, G ; \xi)$ follows if we show that

$$
\sup _{x_{1} \in \mathbf{R}} \int_{\mathbf{R}^{n}} h(|y|)\left|x_{1}-y_{1}\right|^{-\varepsilon v^{\prime}} d y<\infty
$$

To see this, we split the domain of the integration as follows :

$$
\begin{aligned}
\int_{\mathbf{R}^{n}} h(|y|)\left|x_{1}-y_{1}\right|^{-\varepsilon v^{\prime}} d y= & \int_{\left|x_{1}-y_{1}\right|<1} h(|y|)\left|x_{1}-y_{1}\right|^{-\varepsilon v^{\prime}} d y \\
& +\int_{\left|x_{1}-y_{1}\right|>1} h(|y|)\left|x_{1}-y_{1}\right|^{-\varepsilon v^{\prime}} d y \\
= & I_{1}+I_{2}, \quad \text { say. }
\end{aligned}
$$

Clearly $I_{2} \leqslant\|H\|_{1}$. To estimate $I_{1}$ we may assume that $n \geqslant 2$; the case $n=1$ can be easily disposed of since $h$ is bounded. We need further splitting of the domain of the integration. We write $y=\left(y_{1}, y^{\prime}\right), y^{\prime} \in \mathbf{R}^{n-1}$. Then

$$
\begin{aligned}
I_{1} & =\int_{\substack{\left|x_{1}-y_{1}\right|<1 \\
\left|y^{\prime}\right|<1}} h(|y|)\left|x_{1}-y_{1}\right|^{-\varepsilon v^{\prime}} d y+\int_{\substack{\left|x_{1}-y_{1}\right|<1 \\
\left|y^{\prime}\right|>1}} h(|y|)\left|x_{1}-y_{1}\right|^{-\varepsilon v^{\prime}} d y \\
& =I_{3}+I_{4}, \quad \text { say. }
\end{aligned}
$$

It is easy to see that

$$
I_{3} \leqslant\|H\|_{\infty} \int_{|y|<2}\left|y_{1}\right|^{-\varepsilon v^{\prime}} d y<\infty
$$

Next, since $h(|y|) \leqslant h\left(\left|y^{\prime}\right|\right)$

$$
\begin{aligned}
I_{4} & \leqslant \int_{\left|y_{1}\right|<1}\left|y_{1}\right|^{-\varepsilon v^{\prime}} d y_{1} \int_{\left|y^{\prime}\right|>1} h\left(\left|y^{\prime}\right|\right) d y^{\prime} \\
& \leqslant c \int_{\left|y_{1}\right|<1}\left|y_{1}\right|^{-\varepsilon v^{\prime}} d y_{1} \int_{|y|>1} h(|y|) d y<\infty .
\end{aligned}
$$

It remains to estimate $L_{\varepsilon}(G, G ; \xi)$. Note that

$$
L_{\varepsilon}(G, G ; \xi) \leqslant \int_{0}^{\infty} \int_{0}^{\infty} h(r) h(s) r^{n-1} s^{n-1} I_{\varepsilon}(r, s) d r d s
$$

where

$$
I_{\epsilon}(r, s)=\iint_{S^{n-1} \times S^{n-1}}|\langle\xi, r \theta-s \omega\rangle|^{-\varepsilon} \Omega(\theta) \Omega(\omega) d \sigma(\theta) d \sigma(\omega) .
$$


By Hölder's inequality

$$
I_{\varepsilon}(r, s) \leqslant\left(N_{\varepsilon v^{\prime}}(r, s)\right)^{1 / v^{\prime}}\|\Omega\|_{v}^{2}
$$

where

$$
N_{\varepsilon}(r, s)=\iint_{S^{n-1} \times S^{n-1}}\left|r \theta_{1}-s \omega_{1}\right|^{-\varepsilon} d \sigma(\theta) d \sigma(\omega)
$$

Using the estimate (4.3) in (4.2) and then applying Hölder's inequality, we see that

$$
\begin{aligned}
L_{\varepsilon}(G, G ; \xi) & \leqslant c\|H\|_{1}^{2 / v}\|\Omega\|_{v}^{2}\left(\int_{0}^{\infty} \int_{0}^{\infty} N_{\varepsilon v^{\prime}}(r, s) h(r) h(s) r^{n-1} s^{n-1} d r d s\right)^{1 / v^{\prime}} \\
& =c\|H\|_{1}^{2 / v}\|\Omega\|_{v}^{2}\left(\iint_{\mathbf{R}^{n} \times \mathbf{R}^{n}} h(|x|) h(|y|)\left|x_{1}-y_{1}\right|^{-\varepsilon v^{\prime}} d x d y\right)^{1 / v^{\prime}} .
\end{aligned}
$$

Therefore, the desired estimates follows again from (4.1). This completes the proof. $\square$

For a non-negative function $\Omega$ on $S^{n-1}$ we define a non-isotropic Hardy-Littlewood maximal function

$$
M_{\Omega}(f)(x)=\sup _{r>0} r^{-n} \int_{|y|<r}|f(x-y)| \Omega\left(|y|^{-1} y\right) d y
$$

To prove Theorem 2 we also need the following (see Duoandikoetxea [4]).

LEMmA 4. If $\Omega \in L^{q}\left(S^{n-1}\right), q \geqslant 2$, and $w \in A_{2 / q^{\prime}}$, then $M_{\Omega}$ is bounded on $L_{w}^{2}$

Now we can prove Theorem 2. As in Stein [10, pp.63-64], we can show that

$$
\sup _{t>0}\left|\psi_{t} \star f(x)\right| \leqslant c M_{\Omega}(f)
$$

So, by Lemma 4 we see that the condition (1.5) holds for $\psi$ of Theorem 2 with $s=2 / q^{\prime}$.

Next, applying Lemma 3, we see that $J_{\varepsilon}(\psi)<\infty$ for $\varepsilon<\min \left(1 / u^{\prime}, 1 / q^{\prime}\right)$ (note that $h(r)$ of Theorem $2(3)$ is bounded for $r \geqslant 1)$. Combining these facts with the assumption in Theorem 2 (1), we can apply Proposition 2 to reach the conclusion.

Finally, we give the proof of Theorem 3. Clearly $B_{1}(\psi)<\infty$, and $J_{1 /\left(2 q^{\prime}\right)}(\psi)<\infty$ by applying Lemma 3 suitably. Therefore, the conclusion follows from Proposition 2 if we show that the condition (1.5) holds with $s=2 / q^{\prime}$. But, for $q>2$ this is a consequence of the inequality

$$
\sup _{t>0}\left|\psi_{t} \star f(x)\right| \leqslant c M\left(|f|^{q^{\prime}}\right)^{1 / q^{\prime}}
$$


where $M$ denotes the Hardy-Littlewood maximal operator. (This inequality is easily proved from Hölder's inequality.)

To prove condition (1.5) when $q=2$ and $w \in A_{1}$, we may assume that $\psi$ is supported in $\{|x|<1\}$. Then by Schwarz's inequality

$$
\left|\psi_{t} \star f(x)\right|^{2} \leqslant t^{-n}\|\psi\|_{2}^{2} \int_{|y|<t}|f(x-y)|^{2} d y .
$$

Integrating with the measure $w(x) d x$ and using a property of the $A_{1}$-weight function, we get

$$
\begin{aligned}
\int\left|\psi_{t} \star f(x)\right|^{2} w(x) d x & \leqslant\|\psi\|_{2}^{2} \int|f(y)|^{2} t^{-n} \int_{|x-y|<t} w(x) d x d y \\
& \leqslant C_{w}\|\psi\|_{2}^{2} \int|f(y)|^{2} w(y) d y
\end{aligned}
$$

uniformly in $t$. From this the desired inequality follows.

\section{Applications}

It is to be noted that Theorem 1 can be applied to study the $L_{w}^{p}$-boundedness of generalised Marcinkiewicz integrals.

Corollary 1. For $\varepsilon>0$, let

$$
\psi(x)=|x|^{-n+\varepsilon} \Omega\left(x^{\prime}\right) \chi_{(0,1]}(|x|),
$$

where $\Omega \in L^{\infty}\left(S^{n-1}\right)$ and $\int \Omega\left(x^{\prime}\right) d \sigma\left(x^{\prime}\right)=0$. Define a Marcinkiewicz integral

$$
\mu(f)(x)=\left(\int_{0}^{\infty}\left|\psi_{t} \star f(x)\right|^{2} \frac{d t}{t}\right)^{1 / 2} .
$$

Then, the operator $\mu$ is bounded on $L_{w}^{p}$ for all $p \in(1, \infty)$ and $w \in A_{p}$ :

$$
\|\mu(f)\|_{L_{w}^{p}} \leqslant C_{p, w}\|f\|_{L_{w}^{p}}
$$

This result, in particular, removes the Lipschitz condition assumed for $\Omega$ in Stein [9, Theorem 1 (2)].

Next, we consider applications to Carleson measures on the upper half spaces.

Corollary 2. Suppose $\psi \in L^{1}$ satisfies (1.1) and

$$
|\psi(x)| \leqslant c(1+|x|)^{-n-\varepsilon} \quad \text { for some } \quad \varepsilon>0 .
$$


Take $b \in B M O$ and $w \in A_{2}$. Then the measure

$$
d \nu(x, t)=\left|\psi_{t} \star b(x)\right|^{2} \frac{d t}{t} w(x) d x
$$

on the upper half space $\mathbf{R}^{n} \times(0, \infty)$ is a Carleson measure with respect to the measure $w(x) d x$, that is,

$$
\nu(S(Q)) \leqslant C_{w}\|b\|_{B M O}^{2} \int_{Q} w(x) d x
$$

for all cubes $Q$ in $\mathbf{R}^{n}$, where

$$
S(Q)=\left\{(x, t) \in \mathbf{R}^{n} \times(0, \infty): x \in Q, 0<t \leqslant \ell(Q)\right\},
$$

with $\ell(Q)$ denoting the sidelength of $Q$.

This can be proved by using $L_{w}^{2}$-boundedness of the operator $S_{\psi}$ (see Theorem 1) as in Journé [7, Chapter $6 \mathrm{III}, \mathrm{pp} .85-87]$. In [7], a similar result has been proved with an additional assumption on the gradient of $\psi$.

Arguing as in [7, Chapter 6 III, p.87], by Corollary 2 we can get the following.

Corollary 3. Let $\psi$ and $b$ be as in Corollary 2. Suppose $\varphi$ satisfies

$$
|\varphi(x)| \leqslant c(1+|x|)^{-n-\delta}
$$

for $\delta>0$. Then, the sublinear operator

$$
T_{b}(f)(x)=\left(\int_{0}^{\infty}\left|\psi_{t} \star b(x)\right|^{2}\left|\varphi_{t} \star f(x)\right|^{2} \frac{d t}{t}\right)^{1 / 2}
$$

is bounded on $L_{w}^{p}$ for all $p \in(1, \infty)$ and $w \in A_{p}$ :

$$
\left\|T_{b}(f)\right\|_{L_{w}^{p}} \leqslant C_{p, w}\|b\|_{B M O}\|f\|_{L_{w}^{p}} .
$$

Here again we don't need the assumption on the gradient of $\psi$. See Coifman and Meyer [3, p.149] for the $L^{2}$-case.

COROLlary 4. Suppose $\eta \in L^{1}\left(\mathbf{R}^{n}\right)$ satisfies the assumptions of Theorem 1 for $\psi$. Let $\psi, \varphi$ and $b$ be as in Corollary 3 , and define a paraproduct

$$
\pi_{b}(f)(x)=\int_{0}^{\infty} \eta_{t} \star\left(\left(\psi_{t} \star b\right)\left(\varphi_{t} \star f\right)\right)(x) \frac{d t}{t} .
$$

Then, the operator $\pi_{b}$ is bounded on $L_{w}^{p}$ for all $p \in(1, \infty)$ and $w \in A_{p}$ :

$$
\left\|\pi_{b}(f)\right\|_{L_{w}^{p}} \leqslant C_{p, w}\|b\|_{B M O}\|f\|_{L_{w}^{p}} .
$$


Proof: Let $g \in L^{2}\left(w^{-1}\right), w \in A_{2}$. Then, since $w^{-1} \in A_{2}$, by Schwarz's inequality', Theorem 1 and Corollary 3 , for $0<u<v$, we see that

$$
\begin{aligned}
& \left|\iint_{u}^{v} \eta_{t} \star\left(\left(\psi_{t} \star b\right)\left(\varphi_{t} \star f\right)\right)(x) \frac{d t}{t} g(x) d x\right| \\
& \quad \leqslant\left(\iint_{u}^{v}\left|\tilde{\eta}_{t} \star g(x)\right|^{2} \frac{d t}{t} w^{-1}(x) d x\right)^{1 / 2}\left\|T_{b}(f)\right\|_{L^{2}(w)} \\
& \quad \leqslant C_{w}\|b\|_{B M O}\|g\|_{L^{2}\left(w^{-1}\right)}\|f\|_{L^{2}(w)},
\end{aligned}
$$

where $\tilde{\eta}(x)=\eta(-x)$. From this estimate we can see that $\pi_{b}(f)$ is well-defined (see Christ [2, III, Section 3]). Taking the supremum over $g$ with $\|g\|_{L^{2}\left(w^{-1}\right)} \leq 1$, we get the $L_{w}^{2}$-boundedness, and so the extrapolation theorem of Rubio de Francia implies the conclusion. This completes the proof.

See Coifman and Meyer [3, p.149, Proposition 1] for a similar result in the $L^{2}$-case.

\section{REFERENCES}

[1] A. Benedek, A.P. Calderón and R. Panzone, 'Convolution operators on Banach space valued functions', Proc. Nat. Acad. Sci. U.S.A. 48 (1962), 356-365.

[2] M. Christ, Lectures on singular integral operators, Regional conference series in mathematics 77 (Amer. Math. Soc., Providence, R.I., 1990).

[3] R.R. Coifman and Y. Meyer, Au delà des opérateurs pseudo-différentiels, Astérisque 57 (Soc. Math. France, 1978).

[4] J. Duoandikoetxea, 'Weighted norm inequalities for homogeneous singular integrals', Trans. Amer. Math. Soc. 336 (1993), 869-880.

[5] J. Duoandikoetxea and J.L. Rubio de Francia, 'Maximal and singular integral operators via Fourier transform estimates', Invent. Math. 84 (1986), 541-561.

[6] J. Garcia-Cuerva and J.L. Rubio de Francia, Weighted norm inequalities and related topics (North-Holland Publishing Co, Amsterdam, 1985).

[7] J.-L. Journé, Calderón-Zygmund operators, pseudo-differential operators and the Cauchy integral of Calderón, Lecture Notes in Math. 994 (Springer-Verlag, 1983).

[8] J.L. Rubio de Francia, 'Factorization theory and $A_{p}$ weights', Amer. J. Math. 106 (1984), 533-547.

[9] E. M. Stein, 'On the functions of Littlewood-Paley, Lusin, and Marcinkiewicz', Trans. Amer. Math. Soc. 88 (1958), 430-466.

[10] E. M. Stein, Singular integrals and differentiability properties of functions (Princeton Univ, Press, Princeton, N.J., 1970).

Department of Mathematics

Faculty of Education

Kanazawa University

Kanazawa 920-11 Japan

e-mail: shuichi@kenroku.ipc.kanazawa-u.ac.jp 